\title{
Contenido de nutrientes en las raíces finas y el mantillo de rodales de Eucalyptus grandis de diferente edad en la Mesopotamia Argentina
}

\author{
Fine roots and litter nutrient content of Eucalyptus grandis stands presenting \\ different ages in Mesopotamia Argentina
}

\author{
Carolina Pérez $^{\text {a*}}$, Jorge Frangi a, Juan Goya a , Amalia Luy a, Marcelo Arturi ${ }^{a}$ \\ *Autor de correspondencia: ${ }^{\text {a }}$ Universidad Nacional de La Plata, Laboratorio de Investigación de Sistemas Ecológicos y Ambientales \\ (LISEA), LISEA, diagonal 113 N²469, La Plata, Argentina, tel.: +542214271442, perezcarolina9017@gmail.com
}

\begin{abstract}
SUMMARY
Entre Ríos province is an important center of Eucalyptus spp. plantations in Argentina. It was hypothesized that fine root biomass and litter mass increased with age increasing in plantations. Five, seven and seventeen year old stands of Eucalyptus grandis were sampled. All of them were first rotation stands. We estimated the mass of litter and fine roots $(<5 \mathrm{~mm})$ in the top $30 \mathrm{~cm}$ of the soil profile. We determined: carbon, nitrogen, phosphorus, potassium, calcium and magnesium concentrations in living fine roots and litter. Their mineral content was calculated. ANOVA was used for comparisons among stands. Biomass and mineral content of fine roots in the 30 $\mathrm{cm}$ of the soil profile were similar in seven and seventeen year old stands and higher than in the five year old stand. When only $10 \mathrm{~cm}$ of the soil profile were considered, the seventeen year old stand exhibited the highest biomass and root mineral content. Before seven years fine root biomass and mineral content were stabilized. Mass and mineral content of the litter increased with age. Redistribution of roots in the soil profile with stands age might be related to the increased litter stock and its influence on water and nutrient dynamics.
\end{abstract}

Key words: belowground biomass, belowground mineralomass, litter.

\section{RESUMEN}

La provincia de Entre Ríos es un núcleo importante de plantaciones de Eucalyptus spp. en Argentina. Se planteó como hipótesis que con el aumento en la edad de las plantaciones aumenta la biomasa de raíces finas y la masa del mantillo. Se muestrearon rodales de cinco, siete y diecisiete años de edad de Eucalyptus grandis, de primera rotación. Se estimaron las masas del mantillo y de las raíces finas $(<5 \mathrm{~mm})$ en los primeros $30 \mathrm{~cm}$ de profundidad. Se analizaron químicamente: carbono, nitrógeno, fósforo, potasio, calcio y magnesio en raíces finas vivas y en el mantillo. Se calcularon sus contenidos minerales. Se empleó ANDEVA para las comparaciones entre rodales. A $30 \mathrm{~cm}$ de profundidad las raíces finas vivas de los rodales de siete y diecisiete años presentaron similares biomasas y contenidos de nutrientes, pero mayores que a los cinco años. A $10 \mathrm{~cm}$ de profundidad, el rodal de diecisiete años presentó la mayor biomasa y contenido de nutrientes de raíces que aquellos de menor edad. Antes de los siete años se estabilizaron la biomasa y contenido de nutrientes de raíces finas. Con la edad aumentó la masa y contenido mineral del mantillo. La redistribución de raíces en el perfil del suelo con la edad de los rodales se relacionó con el incremento del mantillo y su influencia sobre la dinámica del agua y los nutrientes del suelo.

Palabras clave: biomasa subterránea, contenido de nutrientes subterráneo, mantillo.

\section{INTRODUCCIÓN}

La biomasa subterránea comprende una porción sustancial, 21 a $27 \%$, de la biomasa de los bosques y las raíces finas son consideradas su componente más dinámico (Chapin et al. 2002). Las raíces finas constituyen la vía primaria para la toma de agua y nutrientes, comparables con el rol que cumplen las hojas en la adquisición de carbono y energía lumínica (Jackson et al. 1997). A medida que una plantación crece ocurren cambios en la densidad, biomasa, y contenido de nutrientes de los árboles. En este proceso, el sistema subterráneo resulta fundamental para sostener el crecimiento aéreo (Laclau et al. 2000, Boulliet et al. 2002). A su vez las raíces finas, debido a su elevada tasa de recambio, junto con el mantillo, constituyen un componente clave en el ciclaje de nutrientes (Gordon y Jackson 2000, Bouillet et al. 2002, Nzila et al. 2002, Guo et al. 2006).

La disponibilidad de agua y nutrientes en el suelo presenta un patrón de distribución espacial y temporal heterogéneo al que las plantas responden mediante la proliferación de sus raíces finas en los sitios de mayor disponibilidad de recursos. Este mecanismo constituye una estrategia de fundamental importancia, especialmente en ambientes con baja disponibilidad de nutrientes (Laclau et al. 2001, Hodge 2004).

En plantaciones de Eucalyptus spp. se ha observado que el mantillo influye positivamente en la proliferación superficial de las raíces finas. La acumulación del mantillo se ha asociado con el aumento en la densidad de las raíces en el estrato superior del suelo en respuesta al incremento superficial en las concentraciones de nutrientes en la solución del 
suelo (Laclau et al. 2001, Bouillet et al. 2002). Debido a que el mantillo aumenta con la edad de la plantación es esperable que su efecto sobre la distribución de raíces sea más evidente en las plantaciones de mayor edad.

En la Argentina, las plantaciones de Eucalyptus grandis Hill ex Maiden ocupan aproximadamente 250.000 hectáreas, localizadas principalmente en la Mesopotamia y, en particular, en el noreste de la provincia de Entre Ríos (SAGPYA 2001). Estas plantaciones se han establecido sobre grandes áreas de vegetación nativa, reemplazando con frecuencia pastizales naturales. Estudios al respecto se han centrado sobre el efecto del cambio en el uso de la tierra sobre las propiedades físico químicas de los suelos (Delgado et al. 2006, Jobbágy et al. 2006). Entre los cambios observados, se señala un aumento en la importancia del mantillo como vía de aporte de materia orgánica y ciclaje de nutrientes del suelo (Delgado et al. 2006). La respuesta de las raíces al incremento del mantillo y su efecto sobre la disponibilidad de nutrientes no ha sido estudiada en plantaciones de E. grandis en Entre Ríos.

El objetivo de este estudio fue establecer tendencias de cambio en la masa y distribución en profundidad así como el contenido de nutrientes de raíces finas y del mantillo a lo largo de una cronosecuencia en plantaciones E. grandis de primera rotación implantadas sobre suelos arenosos en el noreste de Entre Ríos. Se plantean como hipótesis que con la edad de la plantación (1) aumenta la biomasa y contenido de nutrientes de las raíces finas en respuesta a un incremento en la biomasa aérea; (2) como resultado de la relación entre la caída y la descomposición, aumenta el almacenaje de materia seca y nutrientes en el mantillo; (3) aumenta la proporción de la biomasa y contenido de nutrientes de raíces finas a menor profundidad, debido al efecto positivo del mantillo sobre la proliferación de raíces en la zona más superficial del suelo mineral.

\section{MÉTODOS}

Área de estudio y sitios de muestreo. El estudio se realizó en las inmediaciones de la ciudad de Concordia, provincia de Entre Ríos (31²3’ S, 58 02’ O). Para el período 19901997 , la temperatura media anual fue de $19^{\circ} \mathrm{C}$; la precipitación media anual alcanzó 1.280,5 mm. Los meses más lluviosos abarcaron desde octubre a abril (Registros Estación Meteorológica INTA-Concordia datos no publicados).

Se muestrearon plantaciones de Eucalyptus grandis de procedencia sudafricana. La ubicación de los sitios de muestreo se basó en la semejanza de los suelos. Se estudiaron tres plantaciones implantadas sobre suelos Oxic Quartzipsamment, Orden Entisol (Soil Survey Staff 1999); estos suelos son conocidos localmente como suelos rojizos arenosos profundos. Se trata de suelos desarrollados sobre materiales muy arenosos, apoyados sobre materiales franco arcillosos rojizos a más de $150 \mathrm{~cm}$ de profundidad, que se han desarrollado a partir depósitos aluviales de un antiguo río Uruguay durante el Plioceno (Aceñolaza 2007). Tasi (2009) ubica estos suelos dentro de la Serie Yuquerí grande, donde el 90 \% de la fracción arena consiste en cuarzo y otros minerales insolubles. Goya et al. (2008) realizaron una descripción de las características de estos suelos (cuadro 1)

Los sitios tenían cinco, siete y diecisiete años de edad al momento de realizarse el muestreo. Las densidades de plantación fueron 1.000 plantas ha $^{-1}$ en las plantaciones de cinco y siete años de edad y de 1.111 plantas ha $^{-1}$ en la de diecisiete años. Los rodales presentaron una superficie entre 5 y 10 ha. Las tres plantaciones estudiadas eran de primera rotación, implantadas sobre pastizales utilizados como pasturas. Las plantaciones recibieron los mismos tratamientos silviculturales: preparación del suelo mediante rastras de discos, plantación con un distanciamiento de $3 \times 3 \mathrm{~m}$, control mecánico de malezas durante los dos primeros años. En ninguno de los rodales se realizaron podas ni raleos y el destino fue la corta final a tala rasa.

Las diferencias en las características estructurales de las plantaciones están en relación con sus edades al momento de los muestreos (cuadro 2).

Raíces. La biomasa de raíces fue estimada por Luy et al. (1997). El muestreo se realizó hasta los $30 \mathrm{~cm}$ de profundidad. Se realizaron cuatro muestreos, correspondientes con cada una de las estaciones del año. En cada fecha y sitio de muestreo se extrajeron quince muestras ubicadas al azar dentro de cada rodal, mediante cilindros de $6,9 \mathrm{~cm}$ de diámetro por $10 \mathrm{~cm}$ de altura a 0-10, 10-20 y 20-30 $\mathrm{cm}$ de profundidad.

La separación de raíces en laboratorio se realizó mediante flotación, tamizado en húmedo y nuevo flotado, la-

Cuadro 1. Características de los suelos Oxic Quartzipsamment (Entisol) ${ }^{1}$. Characteristic of Oxic Quartzipsamment (Entisol) soil.

\begin{tabular}{|c|c|c|c|c|c|c|}
\hline Horizonte & $\begin{array}{l}\text { Profundidad } \\
\text { (cm) }\end{array}$ & $\begin{array}{c}\text { Carbono } \\
\text { orgánico (\%) }\end{array}$ & $\begin{array}{c}\mathrm{CIC} \\
\left(\mathrm{cmol} \mathrm{kg}^{-1}\right)\end{array}$ & $\mathrm{pH}\left(\mathrm{H}_{2} \mathrm{O}\right)$ & $\begin{array}{c}\text { Equivalente } \\
\text { de humedad }(\%)^{2}\end{array}$ & $\begin{array}{c}\text { Saturación de } \\
\text { bases }(\%)^{3}\end{array}$ \\
\hline Ap & $5-16$ & 0,6 & 1,2 & 5,4 & 1,9 & 26 \\
\hline $\mathrm{AC}$ & $28-42$ & 0,4 & 1,0 & 5,6 & 1,7 & 30 \\
\hline
\end{tabular}

${ }^{1}$ Datos extraídos de Goya et al. (2008). Ap: horizonte A perturbado; AC: horizonte de transición entre los horizontes A y C; CIC: capacidad de intercambio catiónico.

${ }^{2}$ Equivalente de humedad: es la cantidad de agua retenida en una muestra de suelo tamizado después que ha sido sometido a una fuerza equivalente a 1.000 veces la de la gravedad (Pritchett 1986).

${ }^{3}$ Saturación de bases evaluada mediante extracción con acetato de amonio 1N (Page 1982). 
Cuadro 2. Datos estructurales de rodales de Eucalyptus grandis de diferente edad ${ }^{1}$.

Structure of Eucalyptus grandis stands of different ages.

\begin{tabular}{lccc}
\hline \multirow{2}{*}{ Variables } & \multicolumn{3}{c}{ Edad del rodal (años) } \\
\cline { 2 - 4 } & 5 & 7 & 17 \\
\hline Área basal $\left(\mathrm{m}^{2} \mathrm{ha}^{-1}\right)$ & 17,5 & 24,0 & 36,6 \\
Altura promedio (m) & 18,6 & 22,6 & 24,3 \\
DAP medio (cm) & 16,0 & 19,0 & 22,5 \\
Densidad (árboles ha-1) & 920 & 840 & 940 \\
IMA (Mg ha-1 año $\left.^{-1}\right)$ & 18,5 & 20,4 & 16,2 \\
Biomasa $\left(\mathrm{Mg} \mathrm{ha}^{-1}\right)$ & & & \\
$\quad$ Total & 92,2 & 142,4 & 274,4 \\
$\quad$ Foliar & 8,1 & 4,2 & 4,1 \\
$\quad$ Ramas & 8,4 & 10,0 & 12,9 \\
$\quad$ Fuste & 75,7 & 128,2 & 257,4 \\
\hline
\end{tabular}

${ }^{1}$ Datos extraídos de Luy et al. (1997).

vado con agua destilada y extracción con pinzas de punta fina. Se consideraron raíces finas a las menores de $5 \mathrm{~mm}$ de diámetro. Las raíces finas se separaron en las categorías vivas y muertas de acuerdo a su textura, flexibilidad y color (Böhm 1979). Se secaron a $70{ }^{\circ} \mathrm{C}$ hasta peso constante. Se pesaron con una precisión de $0,01 \mathrm{mg}$.

Mantillo. En cada uno de los tres rodales se tomaron diez unidades de muestreo de mantillo (horizonte O), de 0,50 $\mathrm{m}^{2}$, distribuidas al azar. Este muestreo se realizó una única vez para cada rodal, entre los meses de mayo y junio de 1997. Estas muestras incluyeron la capa superficial de mantillo suelto (capa L) donde se diferenciaron hojas, ramas, corteza y estructuras reproductivas, y cuando existió, una delgada capa inferior (capa F) de dichos tejidos en mayor estado de degradación y de mayor antigüedad. El material se separó en los siguientes compartimentos: hojas, ramas $<1 \mathrm{~cm}$ de diámetro, ramas $>1 \mathrm{~cm}$ de diámetro, corteza y estructuras reproductivas.

Análisis químico. (1) Para conocer la concentración de nutrientes en los compartimentos del mantillo, el material de cada compartimento perteneciente a las diez unidades de muestreo fue reunido y homogeneizado y de él se extrajeron tres a cinco alícuotas que fueron sometidos a las rutinas analíticas. Así se obtuvo para cada rodal un solo valor de concentración de nutrientes por compartimento, calculado como el promedio de las alícuotas compuestas analizadas. (2) Con las raíces vivas, de cada rodal y estación del año, extraídas del rango entre 0 y $30 \mathrm{~cm}$ de profundidad, se conformaron muestras compuestas; de ellas se extrajeron alícuotas para análisis resultando un total de treinta y seis concentraciones por cada uno de los seis nutrientes (cuatro estaciones por tres rodales por tres alícuotas). Las alícuotas de cada compartimento de las raíces y mantillo fueron secadas en estufa a $70{ }^{\circ} \mathrm{C}$ hasta peso constante, molidas con molino tipo Wiley, tamizadas con malla 20 y digeridas en medio ácido $\left(\mathrm{HNO}_{3}\right.$ concentrado y $\mathrm{H}_{2} \mathrm{O}_{2}$ al $30 \%$ ) (Luh Huang y Schulte 1985). Las concentraciones de fósforo, potasio, calcio y magnesio fueron determinadas con un espectrómetro de emisión de plasma Beckman Spectra-Span V. En otras alícuotas se determinó la concentración de carbono y nitrógeno con un LECO CNS-2000, mediante el método de combustión seca (Tabatabai y Bremner 1991). La precisión se verificó analizando una muestra certificada estándar de hojas de Citrus del National Bureau of Standards (EE.UU.) por cada conjunto de treinta muestras. Adicionalmente, un duplicado de cada décima muestra fue analizado para verificar la consistencia de los resultados.

Contenido de nutrientes. Se obtuvo como el producto de la concentración de cada elemento mineral por la masa del correspondiente compartimento distinguido en la biomasa subterránea y el mantillo.

Análisis estadístico. Debido a que solo se dispuso de un rodal por edad, no existieron repeticiones para ese factor. Los análisis estadísticos se llevaron a cabo utilizando como repeticiones a las observaciones realizadas dentro de cada rodal que en realidad constituyen pseudoréplicas (Hurlbert 1984). En consecuencia, esos análisis estadísticos evaluaron las diferencias entre rodales antes que entre edades. $\mathrm{La}$ masa total del mantillo y de cada compartimiento se comparó entre rodales mediante ANDEVA de una vía tomando a las diez determinaciones realizadas dentro de cada rodal como repeticiones. La masa de raíces y su contenido de nutrientes se analizaron mediante ANDEVA con dos factores intrasujeto (estación del año y profundidad) y un factor entre sujetos (rodal) (von Ende 2001). Se evaluaron los efectos principales, rodal y profundidad y su interacción. Las interacciones rodal por estación y profundidad por estación se evaluaron solamente para comprobar si las diferencias entre rodales y profundidades fueron similares a lo largo del año. Se realizó un análisis a posteriori de las diferencias entre medias de rodales mediante la prueba de Tukey. Las concentraciones de nutrientes en raíces se compararon mediante ANDEVA con un factor intrasujeto (estación del año) y uno entre sujetos (rodal), con una comparación entre medias de rodales a posteriori mediante la prueba de Tukey. Los contenidos minerales de los compartimientos del mantillo no se analizaron estadísticamente debido a que existió un único valor de concentración para cada compartimiento.

\section{RESULTADOS}

Raíces. La masa total de raíces finas (vivas + muertas) hasta $30 \mathrm{~cm}$ de profundidad fue significativamente menor en el rodal de cinco años $\left(1,9 \pm 0,3 \mathrm{Mg} \mathrm{ha}^{-1}\right)$ que en el de siete años $\left(2,9 \pm 0,3 \mathrm{Mg} \mathrm{ha}^{-1}\right)$, y fue similar entre los de siete $\mathrm{y}$ diecisiete años $\left(2,8 \pm 0,1 \mathrm{Mg} \mathrm{ha}^{-1}\right)$. Las diferencias entre rodales y profundidades en la biomasa de raíces finas fueron 
similares entre estaciones ya que no se observaron interacciones con este último factor (rodal por estación: $\mathrm{F}=0,58$, $P=0,74$, profundidad por estación: $\mathrm{F}=0,72, P=0,60$ ). La biomasa de raíces finas vivas representó 70 a $90 \%$ de la masa total de raíces finas (vivas + muertas), dependiendo del rodal y la fecha de muestreo. La biomasa de raíces finas vivas fue similar entre el rodal de siete años $(2,4 \pm 0,2 \mathrm{Mg}$ $\left.\mathrm{ha}^{-1}\right)$ y el de diecisiete años $\left(2,2 \pm 0,2 \mathrm{Mg} \mathrm{ha}^{-1}\right)$ y significativamente mayor que en el de cinco años $(1,5 \pm 0,1 \mathrm{Mg}$ ha $\left.^{-1}\right)(F=16,00, P<0,0001)$. Las diferencias entre profundidades en la biomasa de raíces finas dependieron del rodal analizado ya que se observó una interacción significativa entre los factores rodales y profundidad ( $F=13,29, P<$ $0,0001)$. En el estrato de $0-10 \mathrm{~cm}$ de profundidad la biomasa de raíces finas vivas fue significativamente mayor en el rodal de diecisiete años que en los de cinco y siete años (figura 1). La biomasa de raíces varió significativamente con la profundidad. En el rodal de diecisiete años se observó la mayor disminución de la biomasa con la profundidad.

Las concentraciones de nutrientes en las raíces finas vivas no presentaron diferencias entre estaciones del año en ningún caso ( $P>0,1$ en todos los casos). Por este motivo se presentan los promedios de las cuatro estaciones del año. Las diferencias entre rodales dependieron del nutriente considerado (cuadro 3).

El rodal de cinco años presentó menores concentraciones de nitrógeno y fósforo que los de mayor edad; el rodal de siete años mostró menor concentración de calcio que los otros dos y el de diecisiete años fue el que presentó mayor concentración de magnesio (figura 2). El potasio no difirió entre rodales.

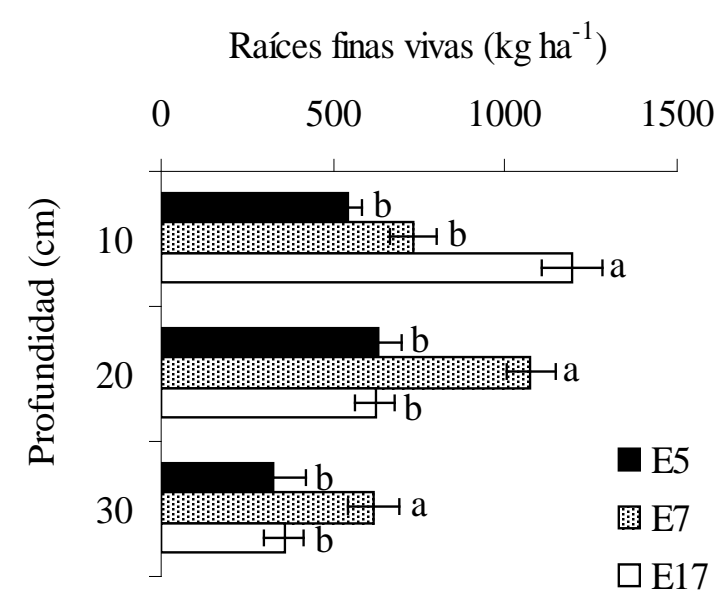

Figura 1. Biomasa de raíces finas por profundidad (media \pm error estándar). E5, E7 y E17: rodales de cinco, siete y diecisiete años de edad, respectivamente. Letras diferentes indican diferencias significativas entre rodales por separado para cada profundidad (Tukey, $P<0,05$ ).

Fine root biomass by depth (mean \pm standard error). E5, E7 and E17: five, seven and seventeen year old stands, respectively. Different letters indicate significant differences among stands within each depth (Tukey, $P<0.05$ ).
Cuadro 3. Resultados de las comparaciones entre rodales de Eucalyptus grandis de diferente edad en la concentración de nutrientes en raíces entre $0-30 \mathrm{~cm}$ de profundidad mediante análisis de varianza.

ANOVA results in comparisons of root nutrient concentrations (0-30 cm depth) among Eucalyptus grandis stands of different ages.

\begin{tabular}{lcc}
\hline Elemento nutritivo & $\mathrm{F}$ & Nivel de significancia \\
\hline Carbono & 5,69 & $*$ \\
Nitrógeno & 7,29 & $*$ \\
Fósforo & 14,74 & $* *$ \\
Potasio & 5,42 & $*$ \\
Calcio & 25,76 & $* *$ \\
Magnesio & 82,84 & $* *$ \\
\hline
\end{tabular}

F: valor de la prueba de Fisher. *: $P<0,05 ; * *: P<0,01$.

Las diferencias entre rodales y profundidades en el contenido de nutrientes de las raíces finas vivas fueron similares en todas las estaciones ya que no se observó interacción entre los factores rodal y estación $(P>0,2$ en todos los casos, excepto para el potasio, $P=0,043$ ), como tampoco entre profundidad y estación para los nutrientes analiza$\operatorname{dos}(P>0,2$ en todos los casos). Analizando los promedios para las cuatro estaciones del año, en el rodal de cinco años se encontraron los menores contenidos de nutrientes de las raíces finas vivas comparados con los restantes rodales, en las tres profundidades analizadas (figura 3). A la profundidad 0-10 cm, los contenidos de todos los nutrientes considerados fueron mayores en el rodal de diecisiete años que en los restantes rodales.

Mantillo. La masa de los compartimentos distinguidos en el mantillo fue mayor en el rodal de diecisiete años que en los rodales de menor edad (figura 4). Como consecuencia la masa total del mantillo también fue significativamente mayor $(\mathrm{F}=10,29, P<0,001)$ en el rodal de mayor edad $\left(13.932 \mathrm{~kg} \mathrm{ha}^{-1}\right)$ que en los de menor edad (7.457 y 10.790 $\mathrm{kg} \mathrm{ha}^{-1}$ en los rodales de cinco y siete años, respectivamente). Las hojas más las ramas menores a $1 \mathrm{~cm}$ de diámetro representaron el $71 \%$, 58 \% y $64 \%$ del total del mantillo en los rodales de cinco, siete y diecisiete años de edad, respectivamente.

En el contenido mineral de los nutrientes analizados se observaron las mismas tendencias que las señaladas en la masa del mantillo (figura 5).

\section{DISCUSIÓN}

La comparación entre rodales de diferente edad permite observar tendencias coincidentes con los cambios esperados para el desarrollo estructural de las plantaciones forestales. A pesar de no disponer de repeticiones para las edades, las diferencias entre sitios pueden interpretarse como debidas a la diferencia de edad en función de la 

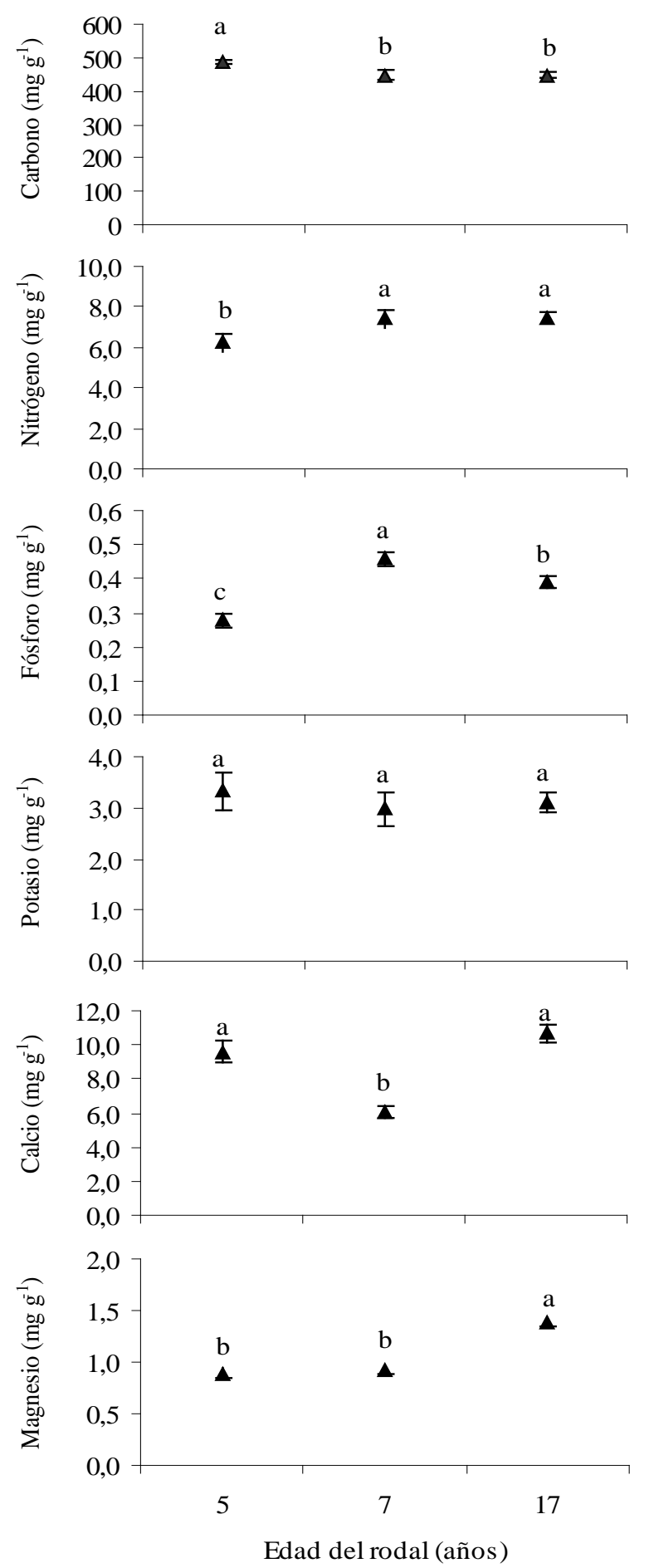

Figura 2. Concentración de nutrientes en raíces finas vivas promedio de los muestreos estacionales y para la profundidad de 0-30 $\mathrm{cm}$. Media \pm error estándar. Letras diferentes indican diferencias significativas entre rodales (Tukey, $P<0,05$ ).

Seasonal average sampling of nutrient concentration in live fine roots at 0-30 $\mathrm{cm}$ depth. Mean \pm standard error. Different letters indicate significant differences among stands (Tukey, $P<0.05$ ).

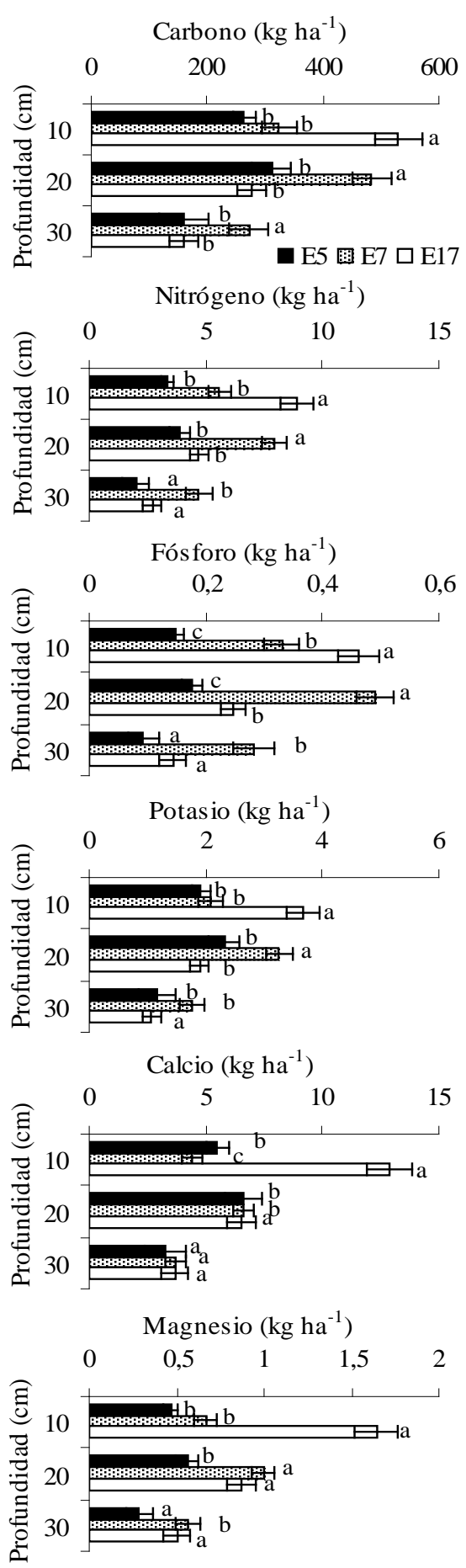

Figura 3. Contenido de nutrientes de raíces finas por profundidad, expresada como promedio de las fechas de muestreo (media \pm error estándar). E5, E7 y E17: rodales de cinco, siete y diecisiete años de edad, respectivamente. Letras diferentes indican diferencias significativas entre rodales por separado para cada profundidad (Tukey, $P<0,05$ ).

Mineral content of fine roots by depth, expressed as an average of the sampling dates (mean \pm standard error). E5, E7 and E17: five, seven and seventeen year old stands, respectively. Different letters indicate significant differences among stands separately for each depth (Tukey, $P<0.05$ ). 


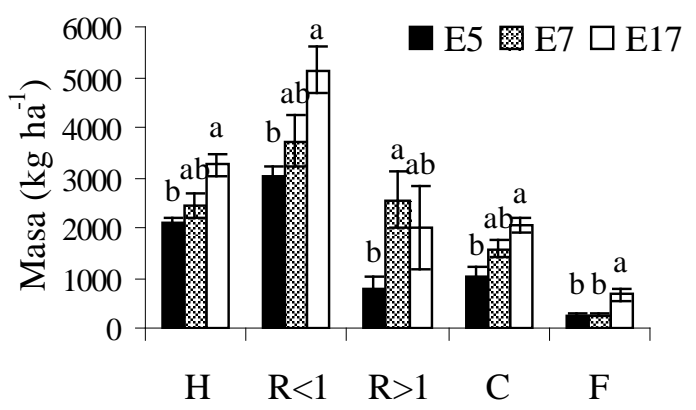

Figura 4. Masa de los compartimentos del mantillo (media \pm error estándar). E5, E7 y E17: rodales de cinco, siete y diecisiete años de edad, respectivamente. $\mathrm{H}$ : hojas, $\mathrm{R}<1$ : ramas $<1 \mathrm{~cm}$, $\mathrm{R}>1$ : ramas $>1 \mathrm{~cm}, \mathrm{C}$ : corteza, $\mathrm{F}$ : frutos. Letras diferentes indican diferencias significativas entre rodales por separado para cada compartimento (Tukey, $P<0,05$ ).

Mass of litter compartments (mean \pm standard error). E5, E7 and E17: five, seven and seventeen year old stands respectively. H: leaves, $\mathrm{R}<1$ : branches $<1 \mathrm{~cm}, \mathrm{R}>1$ : branches $>1 \mathrm{~cm}, \mathrm{C}$ : bark, $\mathrm{F}$ : fruits. Different letters indicate significant differences among stands separately for each compartment (Tukey, $P<0.05$ ).

similitud de las condiciones de clima, suelo, uso previo y manejo de la plantación. La biomasa de raíces finas en todo el perfil estudiado y el mantillo muestran tendencias de aumento con la edad tal como se planteó en las hipótesis (1) y (2). Además, tal como se plantea en la hipótesis (3), la biomasa de raíces finas se concentra en las capas superficiales del suelo con el avance de la edad, ya que en el rodal de diecisiete años representan una mayor proporción del total que en los más jóvenes.

La biomasa de raíces finas vivas y total (vivas + muertas) muestra una tendencia creciente hasta los siete años de plantación, edad a partir de la cual no se observan cambios de stock a lo largo del tiempo. La estabilización de la biomasa subterránea ocurriría antes de los siete años de edad de la plantación. Otros estudios señalan un temprano desarrollo del sistema subterráneo en plantaciones de Eucalyptus spp. (Laclau et al. 2000, Bouillet et al. 2002). Bouillet et al. (2002) estiman un importante incremento de la biomasa de raíces finas en plantaciones de Eucalyptus spp. en el Congo en los primeros dos años de edad, pero no encuentran variaciones entre los dos y nueve años en la cronosecuencia analizada. Dado que las raíces finas constituyen los principales elementos involucrados en la absorción de agua y nutrientes, los cambios en su biomasa podrían vincularse con la demanda de nutrientes de la plantación. La estabilización de la biomasa de raíces finas estaría en relación directa con la estabilización de la biomasa de las hojas, las que constituyen los órganos de los árboles con mayores requerimientos de nutrientes por unidad de biomasa (Grove et al. 1996). En las plantaciones aquí estudiadas se puede constatar que del mismo modo que ocurre con las raíces finas, la biomasa foliar se estabiliza a partir de los siete años (Luy et al. 1997).
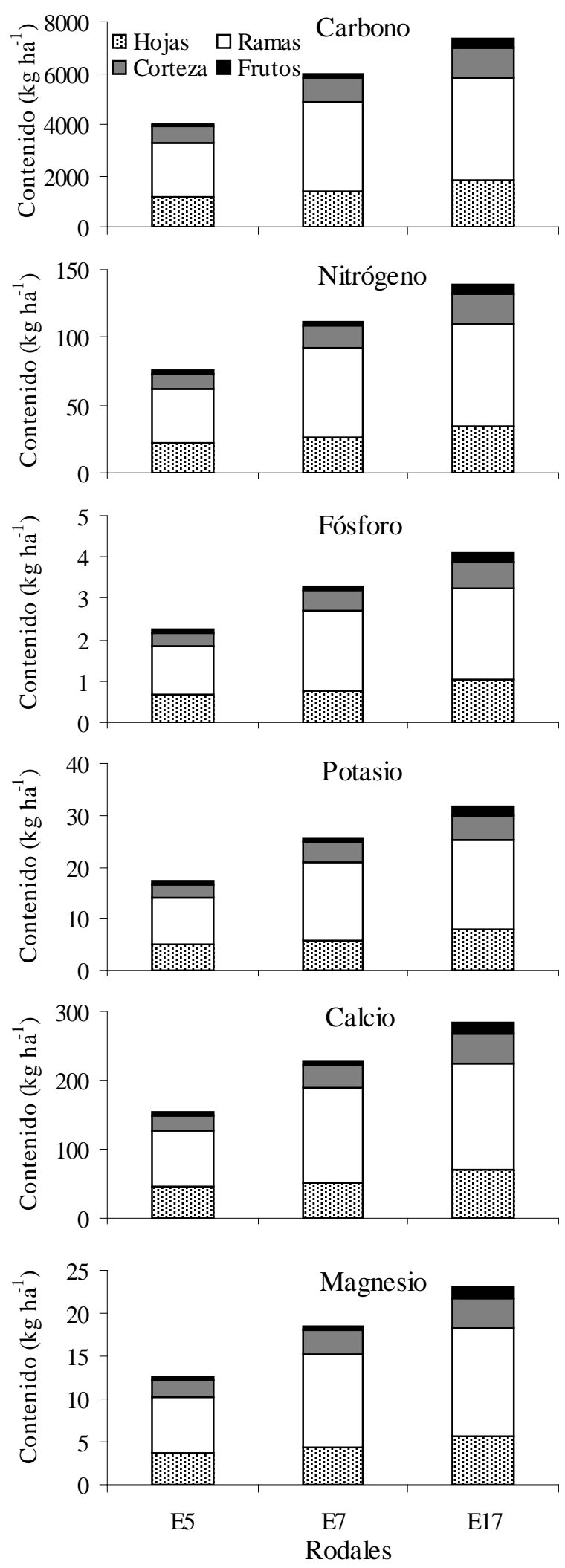

Figura 5. Contenido mineral promedio de los compartimientos del mantillo. E5, E7 y E17: rodales de cinco, siete y diecisiete años de edad respectivamente.

Average mineral content of litter compartments. E5, E7 and E17: stands of five, seven and seventeen years, respectively. 
Aunque la biomasa de raíces finas vivas no varia significativamente entre los siete y los diecisiete años en la totalidad del perfil analizado (0-30 $\mathrm{cm}$ de profundidad), resulta evidente el cambio en su distribución, dado por un incremento de la biomasa en el estrato más superficial del suelo (0-10 cm de profundidad) en la plantación de mayor edad. A su vez, el mantillo también se incrementa a lo largo del tiempo. El aumento de la masa del mantillo con la edad en plantaciones de Eucalyptus spp. es señalado en varios trabajos (Madeira et al. 2002, Goya 2004, Turner y Lambert 2008). La relativamente baja tasa de descomposición del mantillo de Eucalyptus grandis es considerada la principal causa de su acumulación (Goya et al. 2008). Laclau et al. (2001) muestran que las raíces que crecen en el mantillo en plantaciones de Eucalyptus spp. en el Congo captan cantidades significativas de los nutrientes que son liberados por biodegradación del mismo. Sayer et al. (2006) en un bosque tropical de Panamá, hallan una relación inversa entre la biomasa de raíces en el mantillo y en los estratos superficiales del suelo mineral. En bosques húmedos tropicales, la concentración de raíces finas en el mantillo y parte superior de los horizontes minerales es una respuesta para la absorción directa de minerales en un ambiente de bajo déficit de saturación atmosférica y altas precipitaciones (Odum 1970, Bruijnzeel y Veneklaas 1998, Leuschner et al. 2007); en tanto que en condiciones climáticas opuestas, el suministro de nutrientes puede efectuarse a través del bombeo transpiratorio. Laclau et al. (2001) y Sayer et al. (2006) consideran a la masa y dinámica del mantillo como los factores que conducen al cambio en la distribución de las raíces finas. En el presente estudio no fue estudiada la proliferación de raíces en el mantillo, aunque estas están presentes. Sin embargo, el aumento de la biomasa radical en los primeros centímetros del suelo mineral podría responder tanto al aumento del contenido mineral del mantillo con la mayor edad de la plantación como a razones de disponibilidad de agua. Teson (2012) realizó el balance de agua y nutrientes en plantaciones de Eucalyptus grandis en Concordia (provincia de Entre Ríos) de catorce años de edad y estima que la intercepción del mantillo es del $14 \%$ de la precipitación bruta. Como surge del presente trabajo la intercepción aumentaría con la edad de la plantación asociada al aumento del mantillo. En consecuencia, la mayor proporción de raíces en la profundidad de 0-10 cm del suelo en las plantaciones de mayor edad favorecería la captación de agua y nutrientes que pudieran infiltrar a través del mantillo.

Los resultados obtenidos en este trabajo permiten dimensionar la importancia asociada al subsistema mantillo-capa superficial del suelo. Considerando el objetivo comercial de estas plantaciones, en las cuales se prevé una cosecha a tala rasa y en ocasiones, con quemas de los residuos incluyendo el mantillo, se debe considerar la vulnerabilidad de este compartimento, su importancia en el ciclado de nutrientes y como indicador de mantenimiento de la productividad del sitio.

\section{AGRADECIMIENTOS}

Este trabajo fue realizado en el marco del Convenio de Cooperación de Largo Plazo entre la Universidad Nacional de La Plata y el Instituto Internacional de Dasonomía Tropical (IITF) del USDA Forest Service, Puerto Rico. Agradecemos los comentarios de dos revisores anónimos que contribuyeron a mejorar notablemente este manuscrito.

\section{REFERENCIAS}

Aceñolaza FG. 2007. Geología y recursos geológicos de la Mesopotamia Argentina (Vol. 22). Consejo Nacional de Investigaciones Científicas y Técnicas, Instituto Superior de Correlación Geológica, Universidad Nacional de Tucumán. $160 \mathrm{p}$.

Böhm W. 1979. Methods of Studying Roots Systems. Berlin, Alemania. Springer-Verlag. 188 p.

Bouillet JP, JP Laclau, M Arnaud, TA M'Bou, L Saint-Andre, C Jourdan. 2002. Changes with age in the spatial distribution of roots of Eucalyptus clone in Congo Impact on water and nutrient uptake. Forest Ecology and Management 171: 43-57.

Bruijnzeel LA, EJ Veneklaas. 1998. Climatic Conditions and Tropical Montane Forest Productivity: The Fog Has Not Lifted. Ecology 79: 3-9.

Chapin FS III, PA Matson, HA Mooney. 2002. Principles of Terrestrial Ecosystem Ecology. New York, USA. SpringerVerlag. 436 p.

Delgado S, F Alliaume, F García Préchac, J Hernández. 2006. Efecto de las plantaciones de Eucalyptus sp. sobre el recurso suelo en Uruguay. Agrociencia X: 95-107.

Gordon WS, RB Jackson. 2000. Nutrient concentrations in fine roots. Ecology 81: 275-280.

Goya JF. 2004. Análisis del ciclo de nutrientes en Eucalyptus grandis de dos edades de plantación en el NE de Entre Ríos. Paraná, Entre Ríos, Argentina. Actas XIX Congreso Argentino de la Ciencia del Suelo. $10 \mathrm{p}$.

Goya JF, JL Frangi, CA Pérez, F Dalla Tea. 2008. Decomposition and nutrient release from leaf litter in Eucalyptus grandis plantations on three different soils in Entre Ríos, Argentina. Bosque 29(3): 217-226.

Grove TS, BD Thomson, N Malajczuk. 1996. Nutritional physiology of eucalypts: uptake, distribution and utilization. In Attiwill PM, MA Adams eds. Nutrition of Eucalypts. Australia. CSIRO. p. 77-108.

Guo LB, REH Sims, DJ Horne. 2006. Biomass production and nutrient cycling in Eucalyptus short rotation energy forests in New Zealand: II. Litter fall and nutrient return. Biomass and Bioenergy 30: 393-404.

Hodge A. 2004. The plastic plant: root responses to heterogeneous supplies of nutrients. New Phytologist 162: 9-24.

Hurlbert SH. 1984. Pseudoreplication and the design of ecological field experiments. Ecological monographs 54: 187-211.

Jackson RB, H Mooney, ED Schulze. 1997. A global budget for fine root biomass, surface area, and nutrient contents. Proceedings of the National Academy of Sciences of the United States of America 94: 7362-7366.

Jobbágy EG, M Vasallo, KA Farley, G Piñeiro, MF Garbulsky, MD Nosetto, RB Jackson, JM Paruelo. 2006. Forestación en pastizales: hacia una visión integral de sus oportunida- 
des y costos ecológicos. Agrociencia X: 109-124.

Laclau JP, JP Bouillet, J Ranger. 2000. Dynamics of biomass and nutrient accumulation in a clonal plantation of Eucalyptus in Congo. Forest Ecology and Management 128: 181-196.

Laclau JP, M Arnaud, JP Bouillet, J Ranger. 2001. Spatial distribution of Eucalyptus roots in a deep sandy soil in the Congo: relationships with the ability of the stand to take up water and nutrients. Tree Physiology 21: 129-136.

Leuschner C, G Moser, C Bertsch, M Röderstein, D Hertel. 2007. Large altitudinal increase in tree root/shoot ratio in tropical mountain forests of Ecuador. Basic and Applied Ecology 8: 219-230.

Luh Huang CY, EE Schulte. 1985. Digestion of plant tissue for analysis by ICO emission spectroscopy. Communications in Soil Science and Plant Analysis 16: 943-958.

Luy A, JF Goya, JL Frangi. 1997. Distribución de la biomasa aérea y subterránea en plantaciones de Eucalyptus grandis de distintas edades en la región de Concordia, Entre Ríos, Argentina. Actas II Congreso Forestal Argentino y Latinoamericano. $10 \mathrm{p}$.

Madeira MV, A Fabião, JS Pereira, MC Araújo, C Ribeiro. 2002. Changes in carbon stocks in Eucalyptus globulus Labill. plantations induced by different water and nutrient availability. Forest Ecology and Management 171: 75-85.

Nzila JD, JP Boullet, JP Laclau, J Ranger. 2002. The effects of slash management on nutrient cycling and tree growth in Eucalyptus plantations in the Congo. Forest Ecology and Management 171: 209-221.

Odum HT. 1970. Rain forest structure and mineral-cycling homeostasis. In Odum HT, RF Pigeon eds. A Tropical Rain Forest. Virginia, USA. United States Atomic Energy Commission, National Technical Information Service. p. H3-52.

Page LA. 1982. Methods of soil analysis. Part 2, N9. Madison, USA. American Society of Agronomy. 1159 p.
Pritchett WL. 1986. Suelos forestales. México, México. Limusa. $634 \mathrm{p}$.

SAGPyA (Secretaría de Agricultura, Ganadería y Pesca, AR). 2001. Sector Forestal. Dirección de Forestación. Buenos Aires, Argentina. Secretaría de Agricultura, Ganadería, Pesca y Alimentación. 85 p.

Sayer E, E Tanner, A Cheesman. 2006. Increased litterfall changes fine root distribution in a moist tropical forest. Plant and Soil 281: 5-13.

Soil Survey Staff. 1999. Soil Taxonomy: A basic system of soil classification for making and interpreting soil surveys, 2nd edition. Agricultural Handbook 436. Washington DC, USA. Natural Resources Conservation Service, USDA. 869 p.

Tabatabai MA, JM Bremner. 1991. Automated instruments for determination of total carbon, nitrogen, and sulfur in soils by combustion techniques. In Smith KA ed. Soil Analysis Modem Instrumental Techniques Second Edition. New York, USA. Marcel Dekker. p. 261-286.

Tasi HAA. 2009. Aplicación de las Cartas de Suelos de Entre Ríos, Argentina, para evaluar índices de productividad específicos para los principales cultivos agrícolas. Tesis Doctoral. La Coruña, España. Universidad de La Coruña, España. $592 \mathrm{p}$.

Teson N. 2012. Balance hidrológico y flujo de nutrientes asociados al agua en plantaciones de Eucalyptus grandis, en Concordia (Entre Ríos). Tesis Doctoral. La Plata, Argentina. Universidad Nacional de La Plata. 171 p.

Turner J, MJ Lambert. 2008. Nutrient cycling in age sequences of two Eucalyptus plantation species. Forest Ecology and Management 255: 1701-1712.

Von Ende CN. 2001. Repeated-measures analysis: growth and other time-dependent measures. In Scheiner SM, J Gurevicht eds. Design and analysis of ecological experiments. New York, USA. Oxford University Press. p. 134-157.

Recibido: 12.07 .13

Aceptado: 01.10.13 